\title{
SEGMENTATION AND EDGE DETECTION BASED ON MODIFIED ANT COLONY OPTIMIZATION FOR IRIS IMAGE PROCESSING
}

\author{
Abbas Biniaz ${ }^{1}$ and Ataollah Abbasi ${ }^{2}$ \\ ${ }^{1}$ M.Sc. Student, Computational Neuroscience Laboratory, Department of Biomedical Engineering, \\ Faculty of Electrical Engineering, Sahand University of Technology, Tabriz, Iran. \\ abbass_biniaz@yahoo.com \\ ${ }^{2}$ Assistant professor, Computational Neuroscience Laboratory, Department of Biomedical Engineering, \\ Faculty of Electrical Engineering, Sahand University of Technology, Tabriz, Iran. \\ ata.abbasi@sut.ac.ir
}

\begin{abstract}
Ant colony optimization (stocktickerACO) is a meta-heuristic algorithm inspired by food searching behavior of real ants. Recently stocktickerACO has been widely used in digital image processing. When artificial ants move in a discrete habitat like an image, they deposit pheromone in their prior position. Simultaneously, vaporizing of pheromone in each iteration step avoids from falling in the local minima trap. Iris recognition because of its great dependability and non-invasion has various applications. simulation results demonstrate stocktickerACO algorithm can effectively extract the iris texture. Also it is not sensitive to nuisance factors. Moreover, stocktickerACO in this research preserves details of the various synthetic and real images. Performance of ACO in iris segmentation is compared with operation of traditional approaches such as canny, robert, and sobel edge detections. Experimental results reveal high quality and quite promising of stocktickerACO to segment images with irregular and complex structures.
\end{abstract}

\section{Introduction}

Biometric systems have been recently developed by both secluded entities and government agencies. This is due to increase or replace conservative security schemes. Iris is commonly recognized as a reliable biometric measurement [1-3]. Iris in every person is unique and two individuals don't have the same iris. Furthermore, two individual's irises are dissimilar because of their complicated texture structure. In comparison with other biometrics such as finger prints, voice, face, gait, signature, and so on, iris has distinctive characteristics. iris pattern recognition has a rather short history of usage compared with other biometric systems [4-6].
From anatomical point of view, the front seventy percent of the iris encompasses more blood vessels. Various kinds of nerves have been stretched all over the iris. Therefore information easily in a straight line can be transferred to the iris from brain and other structures. Hence, changes in inner body situations and the internal hormone levels lead to iris rapid reaction. Therefore, visible variations in iris's appearance are created. These visible changes contain freckles, pigmentation, appearance white rings surrounding the cornea or sclera, broken texture, and so on $[7,8]$. Systematic variations in the iris's pattern reflect the internal situations of body organs, one's mood, emotions, and one's personality, etc. Furthermore, iris is a robust unique biometric with a very low False Accept Rate (FAR). Applying iris properties, individuals can be easily 
distinguished; this opinion is verified by large-scale authentication experimentations $[4,8]$.

Image segmentation is the procedure of dividing an image into several homogenous subsections based on characteristics such as image gray levels, intensity gradient, tones, colors, or tissue contrast $[9,10]$. There are no universal solutions for image segmentation. However, for specific classes of segmentation problems many good solutions have been developed [11, 12]. In some methods localizing pupil has been developed with wavelet transform (WT), and iris boundary extracted with a differential integral operator [13]. In another approach, binary morphology and local statistics have been applied in iris segmentation [11]. Geodesic Active Contours (GACs) also has been developed in iris image segmentation [4]. In other method Canny edge detection has been developed by Hough transform to iris recognition [14]. In this paper an effective and robust algorithm based on ant colony optimization developed to iris segmentation.

The rest of this paper is organized as follows: in section 2, the ACO algorithm is reviewed and modified. section 3 highlights potential of proposed method through experimental results. Finally Section 4 summarizes conclusions of this paper.

\section{Artificial ant colony in digital habitat}

Ant colony was introduced in 1991/92 by Marco Dorigo as a computational model, which is called ANT SYSTEM. Vitorino Ramos and Filipe Almeida developed idea of using artificial ant colony algorithm to explore in a digital image [15]. ACO mimics the real ant's manner to find the shortest route between the nest and food source. Communication of ants with each other is done by the means of pheromone trails. Tracing more number of ants on certain path becomes more attractive to the others. Therefore other ants follow the path and deposit their own pheromone on it. Establishment of the shortest route is result of this auto catalytic and collective performance. As shown in Figure 1 , ants foraging is started from their nest in diverse directions simultaneously. Ants move in the shorter path when they return to the nest. This path consumes less time and more pheromone deposited rather than the other paths. The shorter route with high probability is followed by other ants. Similarly other ants deposit their individual pheromones on the detected route. Ants are soon fascinated to this path and the best path from the nest to the food source is established as shown in Figure 1. Furthermore some of the ants search in habitat to find new food sources [15-17].

Discretion and parallel nature of ACO are well appropriated in digital images, because ACO searches smartly and utilizes good characteristics such as positive feedback, robustness, and distributed computations $[8,15]$.

\subsection{Pheromone weighting function}

An individual ant can be stated by its position $r$, and orientation $\theta$ as defined by Chialvo and Millonas in [16]. It is sufficient to specify a transition probability from one cell and orientation $(r, \theta)$ to the next cell $\left(r^{*}, \theta^{*}\right)$ a time step later. The response at a given time is supposed to be autonomous of the previous history of the individuals. Utilizing of a pheromone weighting function, the response function can efficiently be translated into a transition rule among the cells and the relative probabilities of moving a pixel $r$ with pheromone density $\sigma(r)$ can be represented as following [15]:

$$
\mathrm{W}(\sigma)=\left(1+\frac{\sigma}{1+\delta \sigma}\right)^{\beta}
$$

Where $\beta$ controls the degree of randomness by following the gradient of pheromone and it is related to the pheromone density. For slight values of $\beta$ the pheromone concentration does not greatly affect the choice; whereas high concentrations cause ants to track the pheromone gradient with more certainty. The notation $1 / f i$ describes each ant can sense pheromone reduction somewhere at great concentrations which means sensory capacity [15].

\subsection{Ant positioning probability function}

Chialvo and Millonas [16] added a weighting factor $\omega\left(\Delta_{i}\right)$ to equation (1). The weighting factor $\omega\left(\Delta_{i}\right)$ certifies ants have higher probability to walk along the earlier direction. $\Delta_{i}$ is associated with the change in direction at each time step. Obviously, it is the basic movement momentum. Affected by the concentration of pheromone in all the eight neigh- 
a

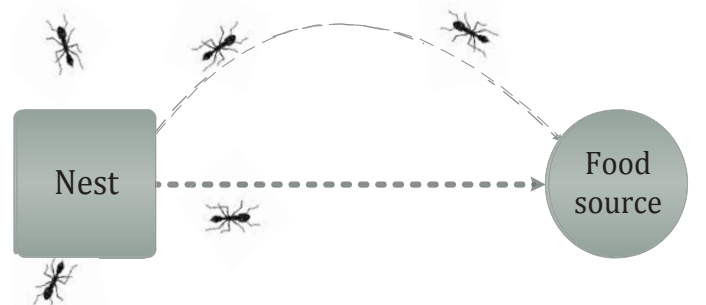

b

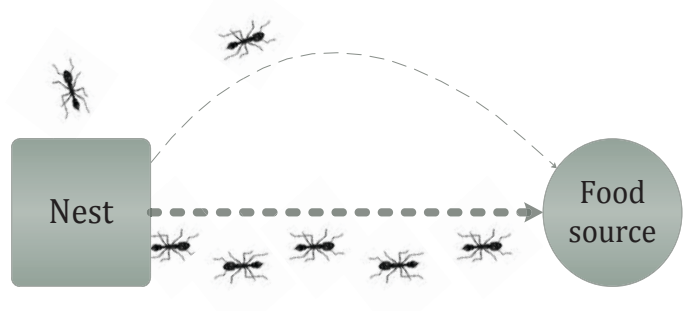

Figure 1. Ants food searching mechanism; (a) searching in several routes (b) establishing optimal route.

boring pixels, each agent can step in one pixel at each time step. When an ant in the lattice goes from left to right, it has the weighing factor $\omega\left(\Delta_{i}\right)$ as depicted in Figure 2.

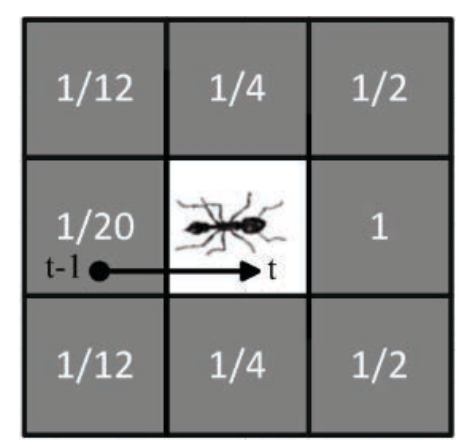

Figure 2. Probability of change in direction for an ant goes from left (time $=\mathrm{t}-1)$ to right $($ time $=\mathrm{t})$ direction.

Normalized transition probability on the lattice to go from pixel $k$ to pixel $i$ at time $t$ is given by [16]:

$$
P_{i k}=\frac{W\left(\sigma_{i}\right) \omega\left(\Delta_{i}\right)}{\sum_{j / k} W\left(\sigma_{j}\right) \omega\left(\Delta_{j}\right)}
$$

The notation $j / k$ specifies the summation of all pixels $j$ in the local neighborhood of $k$. Many ants (percentage of total image pixels) are located on the digital habitat. They are in disorganized situations at start time $t=0$. The probability $P_{i k}$ controls movements of ants among neighborhood cells.

\subsection{Deposition and vaporize of pheromone}

Pheromone deposition method in [16] is controlled by one static parameter, Ramos added a novel dynamic parameter which is not constant and related to a correlation around local neighborhoods. The new pheromone deposition $T$ with two static and dynamic parameters is defined as [18]:

$$
T=\eta+p \Delta_{h}
$$

Where each individual leaves a constant amount $\eta$ of pheromone at the pixel and it is a static parameter situated at every time step $t$. This pheromone decays at each iteration with rate $V . p$ is a constant weighting coefficient and $\Delta_{h}$ is a dynamic parameter to measure similarity degrees between two different lattice windows, including three following terms [18]:

$$
\triangle_{h}=\left\{a \frac{\left|m_{1}-m_{2}\right|}{\operatorname{Max}\left|m_{1}-m_{2}\right|}+b \frac{\left|\sigma_{1}^{2}-\sigma_{2}^{2}\right|}{\left(\operatorname{Max}|| \sigma_{1}^{2}-\sigma_{2}^{2} \mid\right)}+c \frac{S}{S_{\max }}\right\} \cdot(a+b+c)^{-1}
$$

Where $h$ denotes the habitat and the $1^{s t}$ term is responsible for finding differences on overall grey level intensity values. The $2^{\text {nd }}$ term measures differences on windows grey level homogeneity values through variance computations. The $3^{\text {rd }}$ term is computed through differences in two grey level histograms representative of two local neighborhoods and $a+b+c=1 . m_{1}$ is the intensity mean in one lattice window and $\sigma_{1}$ signifies the variance for the same window. $S$ demonstrates the difference of all grey level intensities between two intensity histogram of preceding windows [18].

\subsection{Image segmentation by artificial ant colony}

With the assumption that ants in the system know the "food" in their memory, ACO begins the processing of image segmentation. Therefore they can recognize the pixels which are alike to the 
"food" in the image. The pheromone is deposited in pixels which can affect the motion of the agents. Applying transition rules, the ants change their location in the image at every iteration step. Segmentation results are extracted trough analyzing the pheromone distribution in the image. Details of implementation are described as following:

\subsubsection{Defining food}

Food in ACO is defined as a reference object which can be memorized by the ants. For simplicity an $r$-radius neighborhood $N_{r}(o)$ of a pixel ' $O$ ' in the image is selected. The specified food for ACO in the $i$-th ant's memory at time $t=0$ can be initialized by means of [19]:

$$
\begin{gathered}
F_{i, t=0}=N_{r}(o) \\
N_{r}(o)=\{e \in I \mid\|e-o\|<r\} .
\end{gathered}
$$

I denotes the pixels in the image which should be segmented. Food in the ants' memory, when they find a new food source will be refreshed according to the instructions introduced in the succeeding section.

\subsubsection{Food source finding}

After the reference food definition, ants are responsible to find pixels with analogous properties. Ants are able to compare between the pixels and the specific reference food. For an ant located in the pixel $c$ with the $r$-radius and neighborhood $N_{r}(c)$, the comparison between two different lattice windows is controlled by following [18]:

$$
\begin{gathered}
\mu_{k}(o, c)=\mu_{\varphi}(o, c) \cdot \mu_{\xi}(o, c) \\
\mu_{\varphi}(o, c)=\frac{\min \left(m_{o}, m_{c}\right)}{\max \left(m_{o}, m_{c}\right)}
\end{gathered}
$$

Where $m_{c}$ and $m_{o}$ are meaning of each gray level intensities with $N_{r}(c)$ and $N_{r}(o)$ neighborhood windows; and total texture similarity $\mu_{\xi}(o, c)$ is proposed as follows:

$$
\mu_{\xi}(o, c)=k_{1} p_{j}^{1}+k_{2} p_{j}^{2}+k_{3} p_{j}^{3}+k_{4} p_{j}^{4}+k_{5} p_{j}^{5}
$$

Where $\mathrm{k}_{1}+\mathrm{k}_{2}+\mathrm{k}_{3}+\mathrm{k}_{4}+\mathrm{k}_{5}=1$ and $p_{j}^{d i r}$ is similarity function between two $3 \times 3$ lattice window subimages as follow:

$$
p_{j}^{1}=\frac{1}{1+|f(j)-f(0)|^{\gamma_{1}}} .
$$

The $1^{\text {st }}$ term $p_{j}{ }^{1}$ is set according to the similarity of gray level between the $j$-th neighbor cell $f_{(j)}$ and the center cell $f_{(0)}$. Obviously, it reflects the similarity among neighboring pixels. Similarity between the gray level of the pixels means they belong to the same class.

$$
p_{j}^{2}=\frac{1}{1+|m(j)-m(0)|^{\gamma_{2}}} .
$$

Where $2^{\text {nd }}$ term $p_{j}{ }^{2}$ is set according to the similarity of the gray level means between the $3 \times 3$ neighborhood of $j$-th cell $m_{(j)}$ and the $3 \times 3$ neighborhood of center cell $m_{(0)}$.

$$
p_{j}^{3}=\frac{1}{1+|D(j)-D(0)|^{\gamma_{3}}} .
$$

The $3^{r d}$ term $p_{j}{ }^{3}$ is given by the local texture similarity between the sub-images. Sub-image is the $3 \times 3$ neighborhood of $j$-th cell. $D(j)$ is extended from the $j$-th cell, sub-image variance, and $D(0)$ from the center sub-image variance.

$$
p_{j}^{4}=\frac{1}{1+\left|D_{m}(j)-D_{m}(0)\right|^{\gamma_{4}}} .
$$

The $4^{\text {th }}$ term $p_{j}{ }^{4}$ is given by the local texture similarity between the sub-images. $D_{m}(j)$ is extended from the $j$-th cell sub-image, and $D_{m}(0)$ from the center sub-image. $D_{m}$ is variance of $3 \times 3$ neighborhood when means of all $3 \times 3$ neighborhoods in the image are replaced in center cells of sub-images.

$$
p_{j}^{5}=\frac{1}{1+|d(S(j), S(0))|^{\gamma_{5}}} .
$$

The $5^{\text {th }}$ term $p_{j}{ }^{5}$ like $p_{j}{ }^{3}$ and $p_{j}{ }^{4}$ is given by the local texture similarity between the sub-images. The $d(S(j), S(0))$ is distance between the histogram of two sub-images; sub-image of center cell and one of 8 neighborhood sub-images. $\gamma$ is an experimentally selected balancing parameter. 

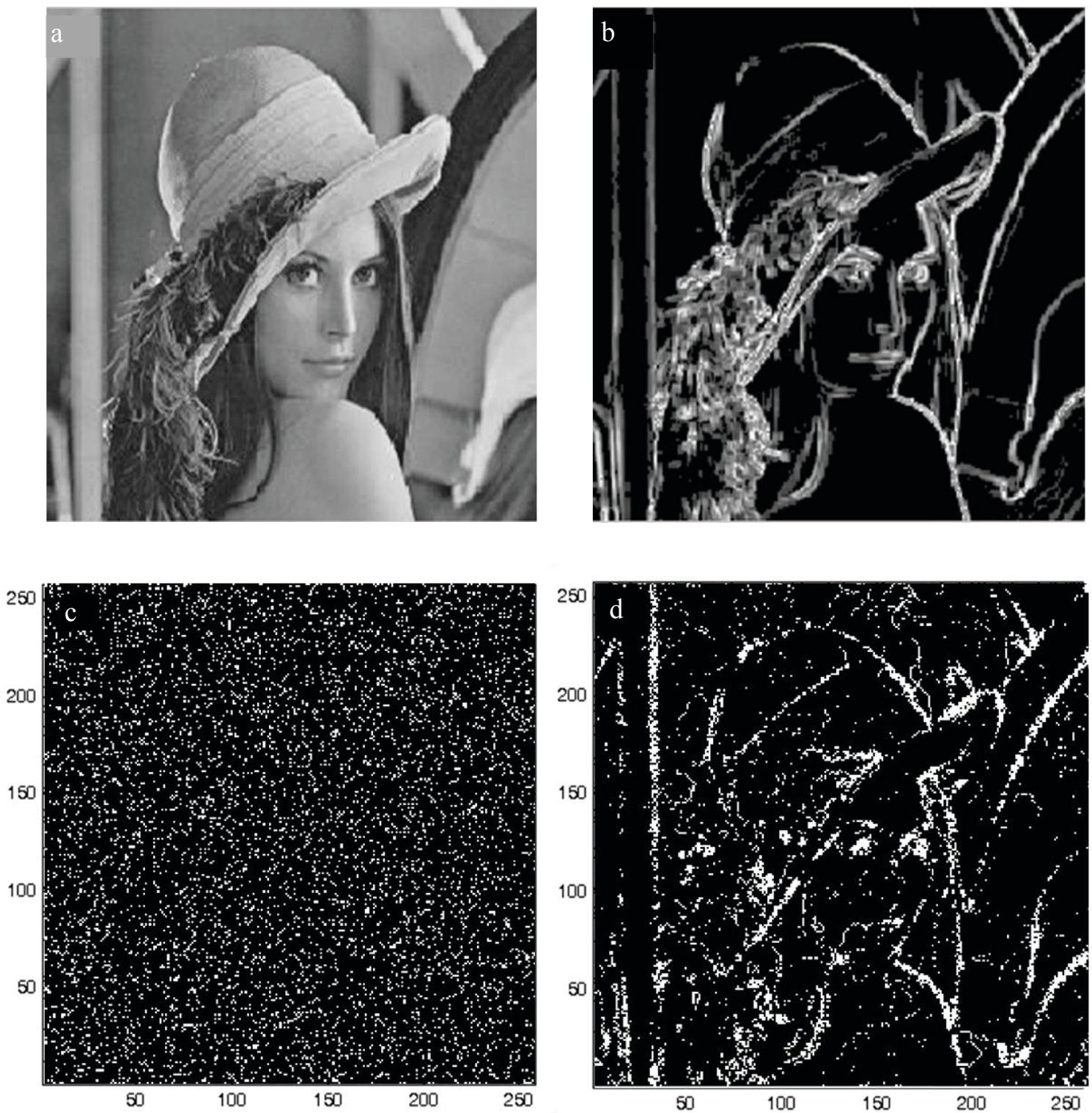

Figure 3. (a) Original image, (b) Performance of ACO after 100 iterations, (c) initial position of ants at digital habitat in first iteration, (d) Position of ants at after 300 iterations when $\beta$ increased to 35 and vaporization is one. 
The pixel $c$ is defined as stimulating the ants searching for a food source when $\mu_{k}(o, c)$ goes beyond a well-defined threshold. The food in the ants' memory at time $t=\tau$ will be refreshed as follow when an ant $i$ consider $c$ as a fresh food source [18]:

$$
F_{i, t=\tau}=a N_{r}(c)+b F_{i, t=\tau-1}
$$

Where $a$ and $b$ are constants.

\subsubsection{Pheromone updating}

As discussed in section 2.4.3 an ant will consider an object as a food source when the value of $\mu_{k}(o, c)$ exceeds from a threshold during the foraging procedure. Each ant has its own threshold $f i$ between 0.4 and 0.9 . Therefore the pheromone deposition $T$ at pixel $c$ can be defined as [19]:

$$
T(c)=\left\{\begin{array}{c}
\eta \stackrel{i f}{\longrightarrow} \mu_{k}(o, c)<\lambda \\
\eta+p \lambda \stackrel{\text { if }}{\longrightarrow} \mu_{k}(o, c) \geq \lambda
\end{array}\right.
$$

Where $\eta$ is a constant amount of pheromone; $p$ is a constant.

\section{Experimental results}

To show the effectiveness of improved ACO, experiments were done on various synthetic and real images. They were filtered by the Canny, Robert, Sobel edge detections, and ACO. Conventional edge detections are implemented by MATLAB toolbox. Figure 3 depicts the performance of ACO technique. Number of agents (ants) in digital habitat are $10 \%$ of total pixels and popular parameters are $\delta=0.02, \beta=3.5, v=0.015, p=1.2, \lambda=0.4$, and $f i=0.07$. Figure $3(a)$ is the original image. performance of ACO is portrayed at Figure 3(b). Initial position of particle swarm at digital habitat is displayed at Figure 3(c). Convergence of agents in digital habitat after 300 iterations for $\beta=35$ and $v=1$ is represented at Figure 3(d).

Pheromone fields in each iteration step improve when similarity between two lattice windows is larger than the defined threshold. Pheromone density intensification depends on the degrees of similarity between two lattice windows. Pheromone field perspective through swarm food searching is portrayed at Figure 4(a)-(c) for different iterations. Pheromone fields are refreshed in each iteration by these experimental values $\delta=0.02, \beta=3.5, v=0.015$, $p=1.2, \lambda=0.4, f i=0.07$ while number of ants is $10 \%$ of the all pixels in the image.

ACO meta-heuristic solves optimization problem in each iteration step by updating and vaporizing of pheromone density. In Figure 5(b)-(d) ACO segmentation development are described after 5, 10 and 50 iteration steps sequentially. Performance of $\mathrm{ACO}$ when number of ants is decreased to $\% 1$ of total pixels and number of iterations increased to 20, 50, 100, and 300 are shown in Figure 5 (e)(h) respectively. These experiments were done by $\delta=0.02, \beta=3.5, v=0.015, p=1.2, \lambda=0.4, f i=0.07$.

To compare performance of ACO with traditional edge detections Figure 6(a) shows a synthetic image [20]. Performance of ACO, ACO with logarithmic transform, Canny, Robert, and Sobel edge detections respectively are shown in Figure 6(b)-(f). obviously the traditional edge detections lost edges however ACO compensate them. Number of ants are $10 \%$ of the all pixels of image.

To scrutinize ACO performance, real iris image has utilized in the experimentations. The output results have comprised with conventional edge detections in Figures 7(d)-(f). Figure 7 shows the results with 100 iteration steps and parameters have been adjusted to $\beta=3.5, v=.015, p=1.2, \eta=0.07$; while number of ants are $10 \%$ of the all pixels. Small values of $\beta$ and large values of $v$ lead to high probability of searching new paths. Hence to deposit further pheromone in digital habitat number of iterations should be increased. Experiment results proved suitable performance of ACO, however it consumes more time than other edge detections as shown in Table 1.

\section{Conclusion}

In this paper an artificial Ant Colony Optimization algorithm was offered to image processing. This approach is inspired by the mechanism of food-searching in the real ant colony. ACO capability to preserve details of the images was investigated in this study on both synthetic and real images. Simulation results by ACO and traditional 

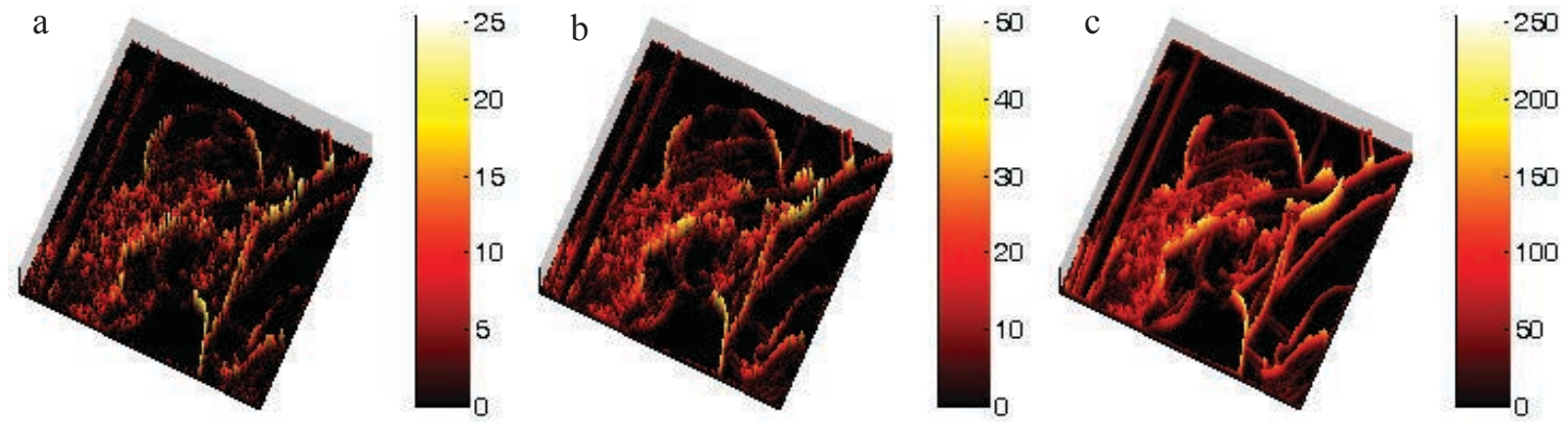

Figure 4. Growing of pheromone fields after (a) 20, (b) 50, (c) 100 iterations.

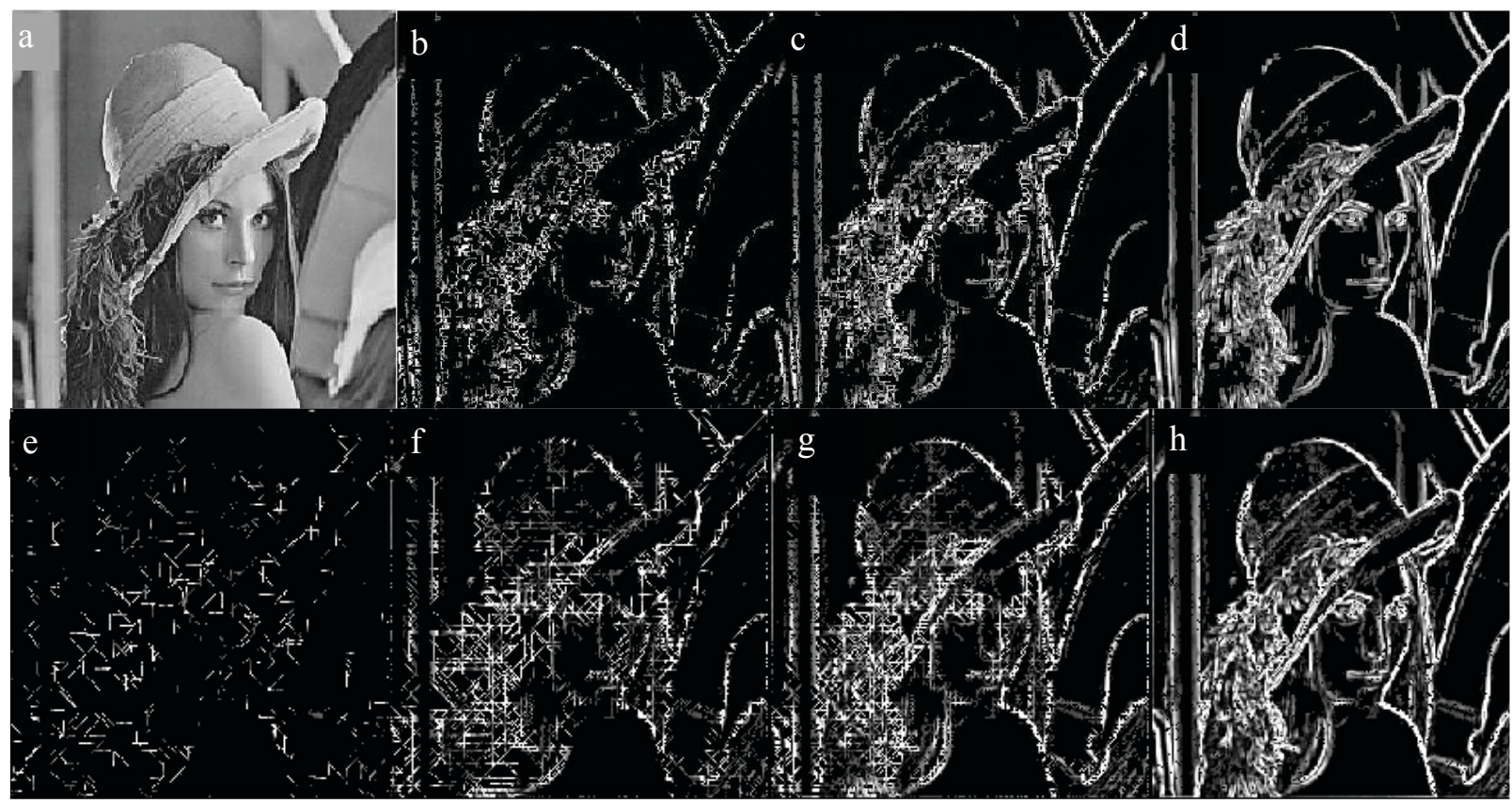

Figure 5. performance of ACO; (a) Original image, (b)-(d) Segmented image after 20, 50, 100 iterations (number of ants is 10\% of total pixels); (e)-(h) performance of ACO after 20, 50, 100 and 300 iteration steps (number of ants is $\% 1$ of total pixels).
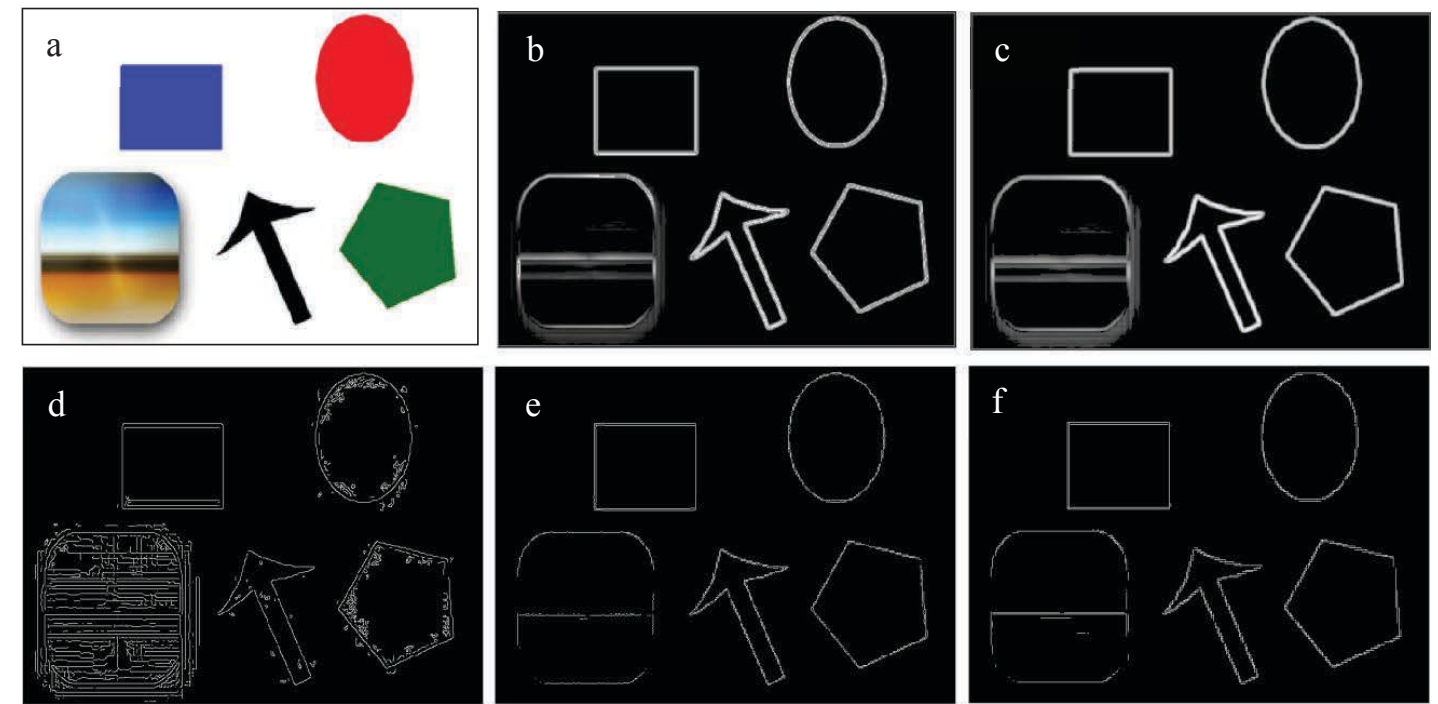

Figure 6. (a)Original image; performance of (b) ACO, (c) ACO with logarithmic transform, (d)Canny, (c)Robert, (f)Sobel edge detections.W8789 

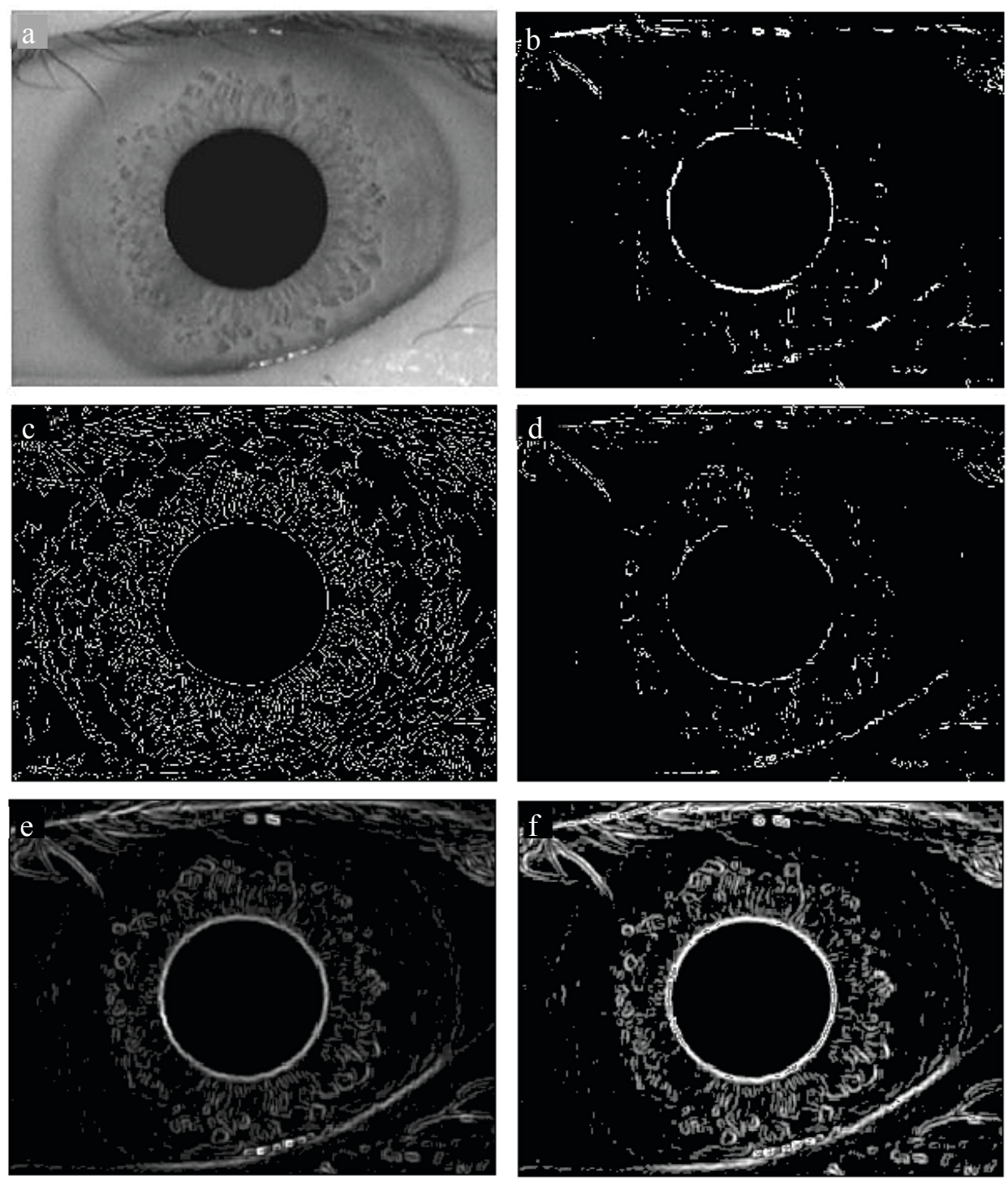

Figure 7. (a) Original iris image; performance of (b) Sobel, (c) Canny, (d) Robert edge detections, (e) ACO and (f) ACO with logarithmic transform.

Table 1. Passed time (in Seconds) by ACO and traditional edge detections.

\begin{tabular}{|l|l|l|l|l|}
\hline Object image & Canny & Robert & Sobel & ACO \\
\hline Synthetic image & 0.283 & 0.862 & 0.080 & 124.72 \\
Lena & 0.084 & 0.051 & 0.048 & 70.04 \\
Iris & 0.266 & 0.084 & 0.078 & 66.05 \\
\hline
\end{tabular}


edge detections showed ACO has superior performance. Furthermore, robustness of ACO against noise was demonstrated on the real iris image. Experimental results revealed efficient performance of ACO in comparison with conventional edge detections however elapsed time by artificial ants is more than these edge detections.

\section{Acknowledgements}

Many thanks to the computational neuroscience laboratory (CNLab) \& ICT research center, Tabriz, Iran, to support this research. The authors also express their sincere gratitude to the anonymous referees for their helpful criticism and valuable suggestions.

\section{References}

[1] P. Khaw, "Iris recognition technology for improved authentication," SANS Institute, 2002.

[2] L. Masek, "Recognition of human iris patterns for biometric identification," M. Thesis, The University of Western Australia, 2003.

[3] R. Bremananth and A. Chitra, "New methodology for a person identification system," Sadhana, vol. 31, pp. 259-276, 2006.

[4] S. Shah and A. Ross, "Iris segmentation using geodesic active contours," Information Forensics and Security, IEEE Transactions on, vol. 4, pp. 824-836, 2009.

[5] X. Liu, K. W. Bowyer, and P. J. Flynn, "Experiments with an improved iris segmentation algorithm," 2005, pp. 118-123.

[6] N. Tripathy and U. Pal, "Handwriting segmentation of unconstrained Oriya text," Sadhana, vol. 31, pp. 755-769, 2006.

[7] D. Cockburn, "A study of the validity of iris diagnosis," The Australian Journal of Optometry, vol. 64, pp. 154-157, 1981.

[8] L. Ma, K. Wang, and D. Zhang, "A universal texture segmentation and representation scheme based on ant colony optimization for iris image processing," Computers \& Mathematics with Applications, vol. 57, pp. 1862-1868, 2009.
[9] M. A. Balafar, A. R. Ramli, M. I. Saripan, and S. Mashohor, "Review of brain MRI image segmentation methods," Artificial Intelligence Review, vol. 33, pp. 261-274, 2010.

[10] R. Kasturi, L. O'gorman, and V. Govindaraju, "Document image analysis: A primer," Sadhana, vol. 27, pp. 3-22, 2002.

[11] W. K. Kong and D. Zhang, "Detecting eyelash and reflection for accurate iris segmentation," International Journal of Pattern Recognition and Artificial Intelligence, vol. 17, pp. 1025-1034, 2003.

[12] M. J. Aligholizadeh, S. Javadi, R. SabbaghiNadooshan, and K. Kangarloo, "An Effective Method for Eyelashes Segmentation Using Wavelet Transform," 2011, pp. 185-188.

[13] Y. Chen, S. Dass, and A. Jain, "Localized iris image quality using 2-D wavelets," Advances in Biometrics, pp. 373-381, 2005.

[14] M. Mahlouji, A. Noruzi, and I. Kashan, "Human Iris Segmentation for Iris Recognition in Unconstrained Environments," 2012.

[15] V. Ramos and F. Almeida, "Artificial ant colonies in digital image habitats-a mass behaviour effect study on pattern recognition," Arxiv preprint cs/0412086, 2004.

[16] D. R. Chialvo and M. M. Millonas, "How swarms build cognitive maps," NATO ASI SERIES F COMPUTER AND SYSTEMS SCIENCES, vol. 144, pp. 439-439, 1995.

[17] T. Niknam, R. Khorshidi, and B. B. Firouzi,"A hybrid evolutionary algorithm for distribution feeder reconfiguration," Sadhana, vol. 35, pp. 139-162, 2010.

[18] H. Cao, P. Huang, and S. Luo, "A novel image segmentation algorithm based on artificial ant colonies," Medical Imaging and Informatics, pp. 63-71, 2008.

[19] P. Huang, H. Cao, and S. Luo, "An artificial ant colonies approach to medical image segmentation," Computer Methods and Programs in Biomedicine, vol. 92, pp. 267-273, 2008.

[20] S. A. Etemad and T. White, "An ant-inspired algorithm for detection of image edge features," Applied Soft Computing, vol. 11, pp. 4883-4893, 2011. 